\title{
Selectivity of SPR fiber sensors in absorptive media: An experimental evaluation
}

\author{
Ivo Leite $^{a}$, María-Cruz Navarrete ${ }^{a}$, Natalia Díaz-Herrera ${ }^{b}$, Agustín González-Cano $^{b, *}$, Óscar Esteban ${ }^{c}$ \\ a Departamento de Óptica, Facultad de CC Físicas, Universidad Complutense, Ciudad Universitaria s/n, 28040 Madrid, Spain \\ b Departamento de Óptica, Escuela Universitaria de Óptica, Universidad Complutense, Arcos de Jalón 118, 28037 Madrid, Spain

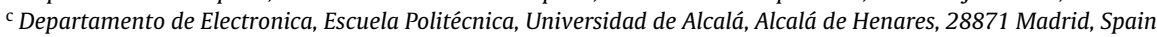

\section{A R T I C L E I N F O}

\section{Article history:}

Received 14 April 2011

Received in revised form 14 July 2011

Accepted 16 August 2011

Available online 24 August 2011

\section{Keywords:}

Surface plasmon resonance

Fiber optic sensors

Absorption

Tapered optical fibers

\begin{abstract}
A B S T R A C T
Conventional surface plasmon resonance (SPR) fiber sensors show selective behavior when resonance wavelengths are tuned to absorption peaks of the surrounding medium, as it is experimentally shown and evaluated in this paper by using a doubly-deposited uniform-waist tapered optical fiber (DLUWT) and a dye as a test material. This behavior is clearly distinguishable from the usual response to nonabsorbing media and can be used to develop a new concept of SPR transducers. At the same time, the obtained results, which are in accordance with the theoretical predictions permit to increase the basic knowledge on surface plasma waves excitation in optical fibers.
\end{abstract}

(c) 2011 Elsevier B.V. All rights reserved.

\section{Introduction}

To say that surface plasmon resonance (SPR) sensors are a most important, almost indispensable tool in chemical and biological analysis and environmental control is no longer a novelty. The field has reached its maturity and a plethora of concepts, commercial devices and laboratory tested setups are depicted in the several reviews published in the literature [1-3]. It would seem impossible to add significant new material to the discussions, especially when dealing with devices conceived for application in real in situ measurements. However, strange as it could appear, there has been surprisingly little study on the behavior of SPR transducers immersed in absorptive media. By the very nature of their working principle, SPR sensors are refractometres and are designed to work with dielectric media, and are also quite local in their detection range. In this context, the only way to achieve a species-selective response of SPR devices is to add some recognizing elements to, through a chemical or biological reaction, capture the desired analyte in the proximity of the sensing region. This implies an additional work of selection of the reagents and the need of a functionalization of the sensor surfaces.

The pressure to obtain better general performance and, in particular, lower detection limits is great, and major efforts in the field have been directed towards this goal. It is in this area where the possibilities of added absorptive components have been invoked as a

\footnotetext{
* Corresponding author. Tel.: +34 91394 6903, fax: +34 913946885

E-mail address: agus@fis.ucm.es (A. González-Cano).
}

powerful tool to enhance the response of the devices [4-8]. Generally speaking, the need is not in those cases to improve and/or achieve selectivity, but to improve responsivity. The absorption does not take place in the analysed media or substance, but in the transducer. And only very rarely the systems proposed in the literature are fiber sensors, despite the many advantages that, in terms of capability, robustness, simplicity and cost offer optical fiber devices. This fact contributes to obscure the analysis on the influence of absorption in the behavior of the plasmon resonances, since most of the usual SPR devices are based in attenuated total reflection and are angularly interrogated, thus losing the crucial information that can only be provided by spectral interrogation, the usual way to proceed with fiber sensors.

Some time ago, the authors showed [9] that, if the plasmon resonance wavelengths of a SPR fiber sensor are tuned to absorption peaks in the surrounding medium, a specific, clearly distinguishable behavior (compared to the usual one) occurs. In that case, theoretical simulations were performed for a conventional SPR fiber sensor immersed in a mixture containing a dye (Rhodamine 6G), one of whose absorption peaks was made coincident to the plasmon resonance wavelength of the sensor. Being plasmon resonance equivalent to an anomalously significant absorption, one could think that the presence of an additional absorption mechanism for the very same wavelength could only contribute to a deepening of the plasmon dips. Quite on the contrary, what the simulations showed is a certain inhibition of the resonance. This is due to the strong variation of the real part of the refractive index that is always associated to a high value of the imaginary part (in fact, what we have there is a region of anomalous dispersion), which destroys 


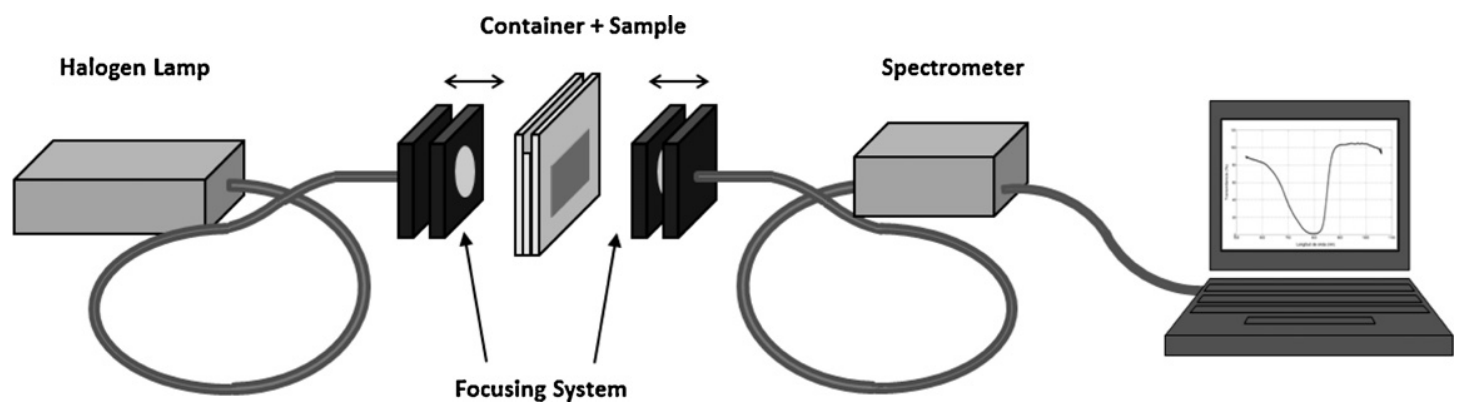

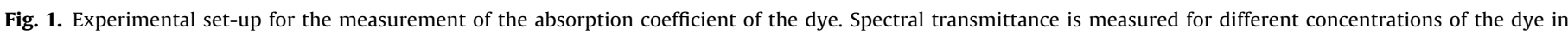
methanol.

the phase matching conditions needed to excite the plasma waves. In this sense, the system reacts in two very different ways to the variations in the refractive index of the tested medium. If we have an increasing in the real part, the plasmon dips move to longer wavelengths. If the change is in the complex part, the plasmon dips become less and less pronounced depending on the concentration of the absorbent species, and they can eventually disappear. This can be used, as it was said in the paper, as the theoretical basis for a new concept of SPR sensor of manifold possibilities, which could be selective without the need of the addition of any recognizing elements, and could provide multi-analyte measurements without the need of complicating the arrangement.

What we show in this work is the experimental confirmation of these predictions and, therefore, the feasibility of the new concept of SPR sensor aforementioned. We have used a most simple setup, well tested fiber devices based on doubly-deposited uniform-waist tapered fibers (DLUWTs) [10-12], and a commercial dye that shows an absorption peak in the region of $800 \mathrm{~nm}$ (a typical value for our plasmon resonance wavelengths). With these results we contribute to an improvement on the knowledge and understanding of the behavior of surface plasma wave excitation in fiber waveguides surrounded by absorbing media, and open the way for the development of compact, versatile, very simple devices that can be designed to be selective to specific analytes.

\section{Characterization of the refractive index of the absorbing medium and theoretical simulations}

As we have said, we have selected a dye with an absorption peak in the region of $800 \mathrm{~nm}$. Given the already proven versatility of DLUWTs, it is really not quite important the specific region in which we work, but it is crucial from the point of view of our method to tune plasmon resonances and absorption wavelengths, so, since we are essentially interested in a proof-of-principle and since our usual working range is the very near IR, we have preferred to look for a substance able to proportionate us well defined absorptions in that area. The selected dye is IR-140, $\mathrm{C}_{39} \mathrm{H}_{34} \mathrm{Cl}_{2} \mathrm{~N}_{3} \mathrm{~S}_{2} \cdot \mathrm{ClO}_{4}$ [13]. We have experimentally measured the imaginary part of the refractive index of the dye solved in methanol for different concentrations, varying the molarity of the solutions between $10^{-4} \mathrm{M}$ and $6 \times 10^{-3} \mathrm{M}$. To do this we have used the experimental setup shown in Fig. 1, based on the determination of the spectral transmittance of the mixture [14]. The light coming from a wide-spectrum halogen lamp is collimated and then passes through a $1 \mathrm{~mm}$ thick glass
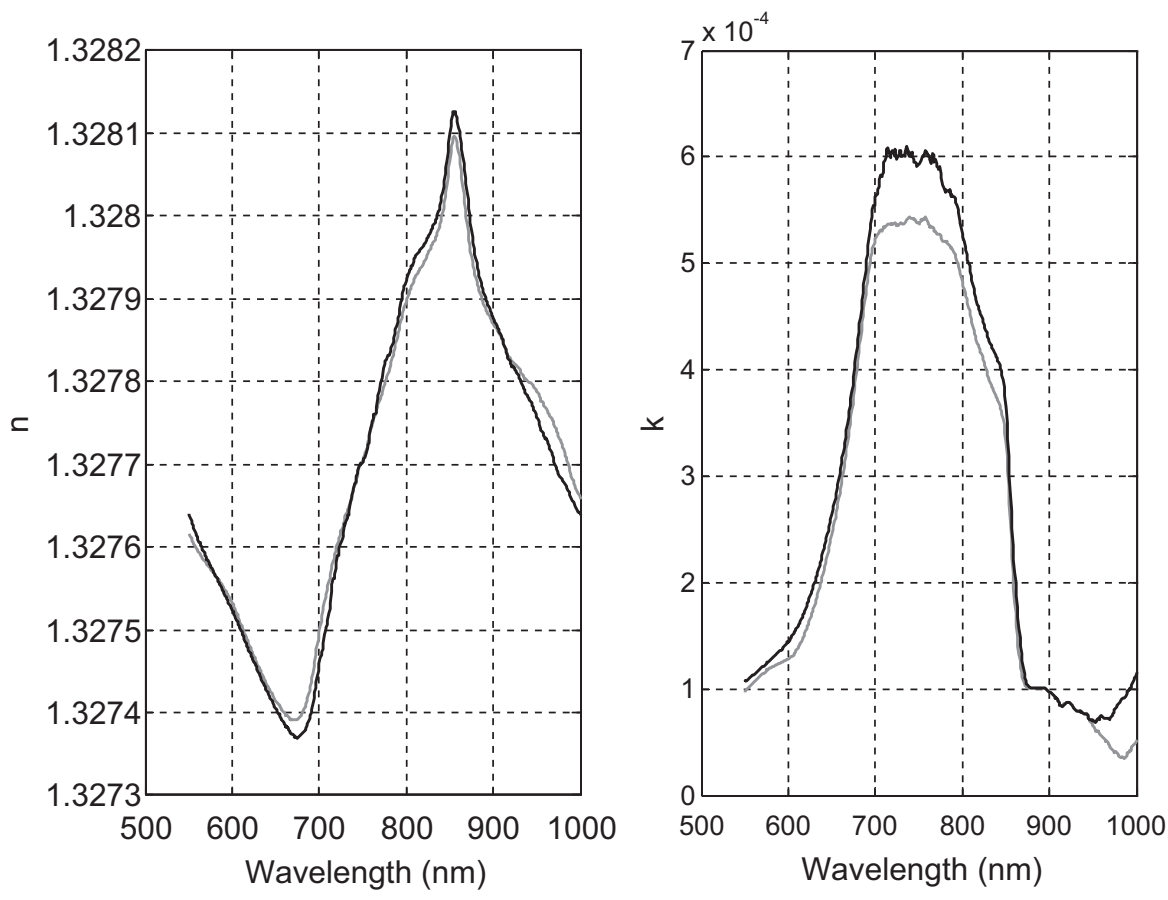

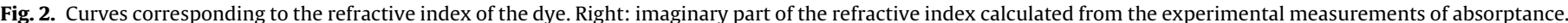

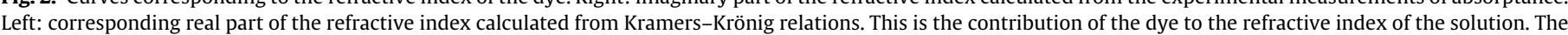
two curves correspond to concentrations of dye of $2.16 \times 10^{-3}$ and $5.9 \times 10^{-3} \mathrm{M}$. 
cuvette containing the sample. Transmitted light is subsequently focused onto a fiber optic connected to a spectrometer Avantes Avalight 2048 with a $1 \mathrm{~nm}$ resolution. Transmittance is then obtained by comparing the output light flux with the reference, consisting of pure methanol. Starting from these data we can easily obtain the spectral absorptance values corresponding to the various concentrations and then the imaginary parts of the refractive indices of the mixtures of methanol and dye. Using Kramers-Krönig relations we can then calculate the real part of the indices. In Fig. 2 we show two representative values of the imaginary part for two different dye concentrations $\left(4.16 \times 10^{-3}\right.$ and $\left.5.9 \times 10^{-3} \mathrm{M}\right)$ and the corresponding contributions of the absorbing species to the real part of the refractive index.

It is important to note that, as expected, an absorption peak is characterized by a maximum of the imaginary part and a region of anomalous dispersion in the real part. As commented above this is the main reason of the variation of the surface plasmon excitation conditions and the basis of our method. Although in this case the values of the imaginary parts are not really too high for the concentrations of dye employed, they are enough to experimentally prove this basis.

To estimate the effect of the presence of the dye in the behavior of the SPR sensors we have performed theoretical simulations starting from these refractive index values. We have used, like we have already done in the past to design the transducers, a quasi-geometrical model [15]: it is the usual matrix formalism for reflective calculations in multilayer structures, taking into account Goos-Hänchen effect. In this case the multilayer is formed by the fiber as substrate and the two layers deposited in the DLUWT, one metallic (aluminum) and the other one dielectric (titanium dioxide). These materials have been commonly used by us in the past and have proven their convenience. We have selected their thicknesses to provide plasmon resonances in the region of $800 \mathrm{~nm}$ for refractive indices of the order of that of methanol (1.3277). In this model, that produces accurate enough results to permit the design of the transducers, the contribution of the absorption appears in the matrix that corresponds to the outer medium, which now includes complex values for the refractive index, instead of the usual real ones. What we have obtained from these calculations is shown in Fig. 3, where only four curves appear, one for the methanol with no dye and the other three for different values of dye concentration. As we can see, the effect of the presence of this dye is a somewhat paradoxical inhibition of the plasmon dip, instead of the common displacement that we can observe, both theoretically and experimentally when the outer medium is a nonabsorbing one.

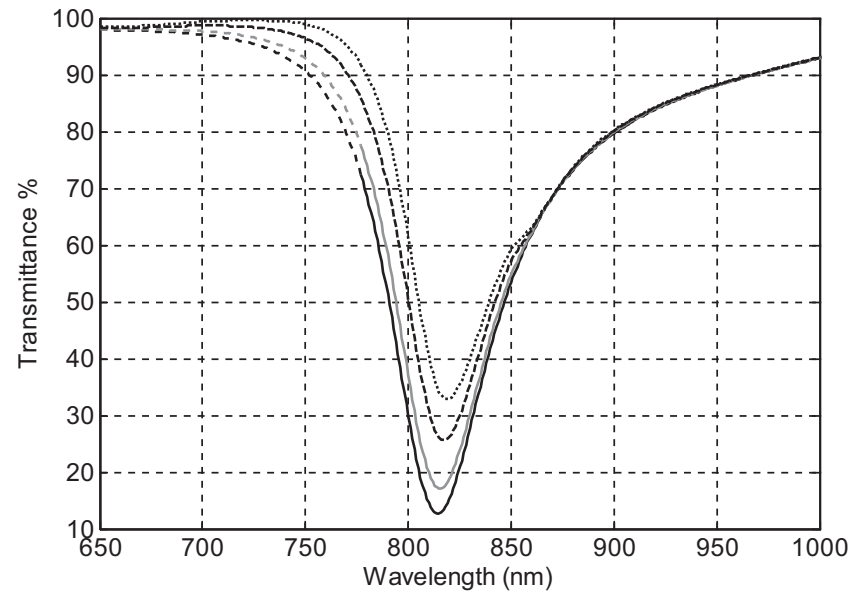

Fig. 3. Simulation curves showing the effect of the presence of the dye in several concentrations in the plasmon dip of the transducer. The curves correspond to pure methanol and concentrations of the dye of $4 \times 10^{-3}, 3 \times 10^{-2}$ and $4.5 \times 10^{-2} \mathrm{M}$.

For the simulations we have postulated a configuration of the transducer that provides a plasmon resonance coincident with the absorption peak. Of course, since the imaginary part of the refractive index of the mixture presents a wide maximum, we have some freedom of choice and it is not too critical to place the plasmon resonance in a specific wavelength, given that we are still within this absorption region. If both absorptions and plasmons are well defined and characterized by narrow peaks/dips the selectivity obtained would improve. But, as it is easy to check in theoretical simulations, if we do not have this coincidence, the effect of a variation in the index of the mixture only produces the usual dip displacement effect. The increase in the minimum transmittance is then due to the presence of the absorbent species and it is more marked when the concentration increases.

\section{Experimental results: behavior of the sensors with the variation of the concentration of the absorbing medium}

We have fabricated the DLUWTs with a travelling burner scheme, where a single-mode fiber optimized for $850 \mathrm{~nm}$ is gently stretched and heated, so a controlled narrowing of the guide is induced. In this way we have a so-called uniform-waist tapered fiber, with very low losses, and therefore we can call this an adiabatic taper $[10,12]$. We have deposited on it asymmetric layers of aluminum $(8 \mathrm{~nm})$ and titanium dioxide $(47 \mathrm{~nm})$. With these

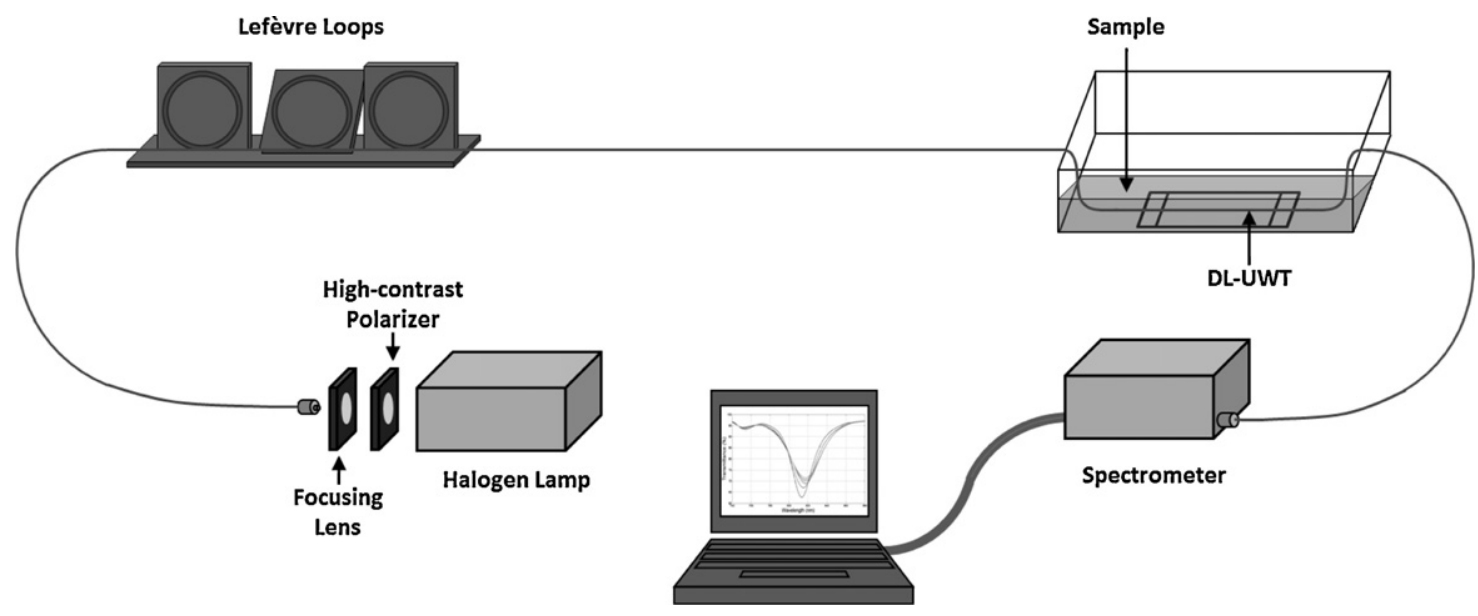

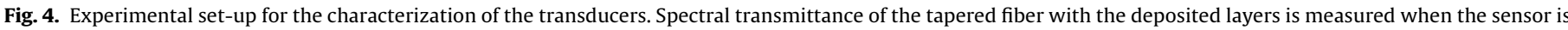
immersed, first in a nonabsorbing solution and then in a solution of methanol with different concentrations of the dye. 


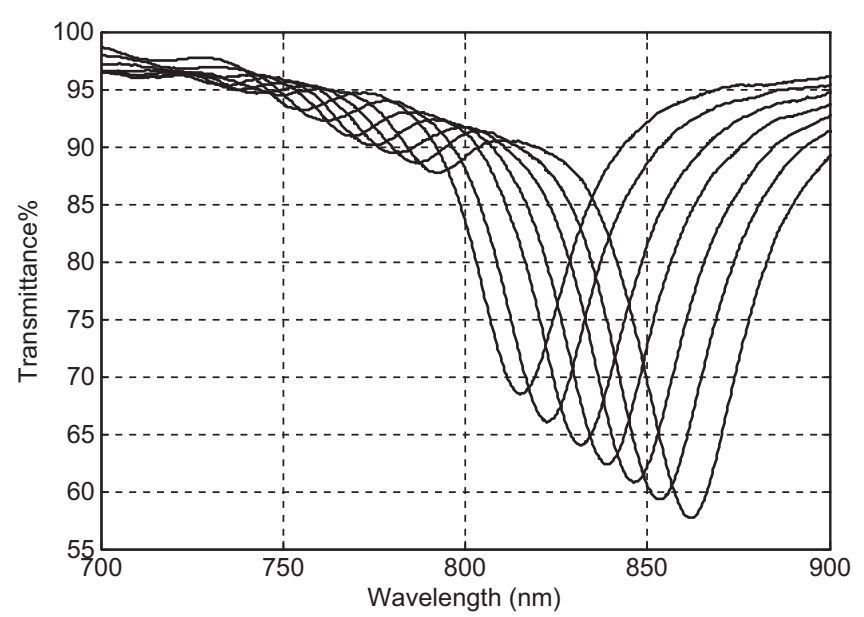

Fig. 5. Experimental results showing the behavior of the sensor when immersed in a mixture of methanol and different quantities of ethyleneglycol, with refractive index varying from 1.3287 to 1.3440 . This medium is nonabsorbing and the plasmon dips behave in the usual way, displacing to the right when the refractive index is increased.

thicknesses of these materials we are able to excite plasmons for the correct range of outer refractive indices in our experiment.

We have characterized the behavior of the devices by measuring their spectral transmittance with the setup shown in Fig. 4. We have used a halogen lamp as light source and a CCD spectrometer Avantes Avalight 2048 as detector.

To test the response of these conventional, nonselective (in principle) elements we have first tested their response when immersed in a nonabsorbing medium. We have mixed methanol with variable quantities of ethylene glycol, thus changing the (real) refractive index of the medium between 1.3287 and 1.3440 . The results obtained are shown in Fig. 5. As we can see, an increase in the index produces a displacement of the dip to the right (towards longer

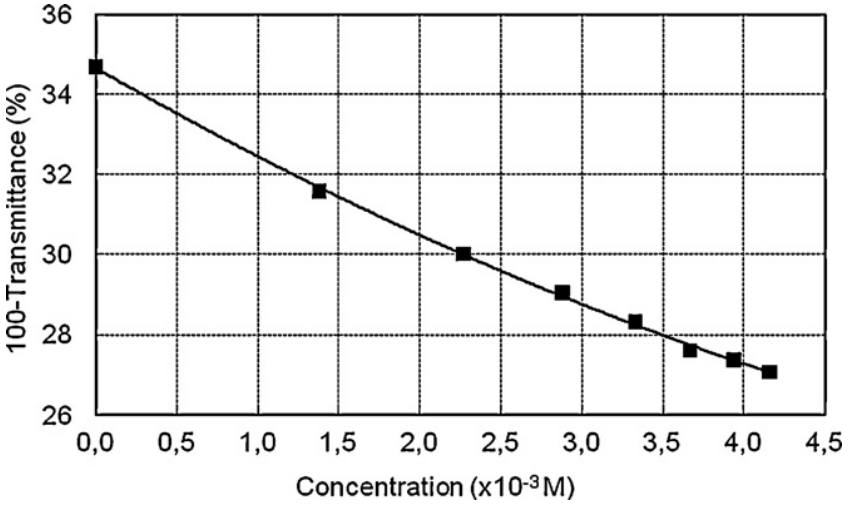

Fig. 7. Effect of the different concentrations of dye in the depth of the plasmon dips. In the $y$-axis we have represented one minus the transmittance associated to the minima, taking this as the significant parameter of the "definition" of the plasmons.

wavelengths) and a slight decrease of the transmittance (i.e., a better definition of the plasmon dip, a deepening of the resonance). This is the common behavior of SPR sensors with nonabsorbing media and this is the basis of SPR chemical and biological sensing. Of course, in this case the response is nonselective: any medium with those indices would produce the same transmittance curves.

In opposition to this we see what happens when the mixture is made of methanol and our absorbing dye IR-140 in Fig. 6 . As we can see there (and especially in the zoomed area that appears in the inset), there is hardly any displacement of the dip to the right and there appears a clear decrease in the definition of the resonances (i.e., an increase in the minimum spectral transmittances). This is the mark of an absorbing medium when there is a tuning between plasmon resonance and absorbing wavelengths. We also see that the dip is less pronounced when the concentration of the dye increases, as predicted in the simulations.

In Fig. 7 we show the evolution of the transmittance for the minima of Fig. 6. To emphasize the effect of "plasmon inhibition" caused

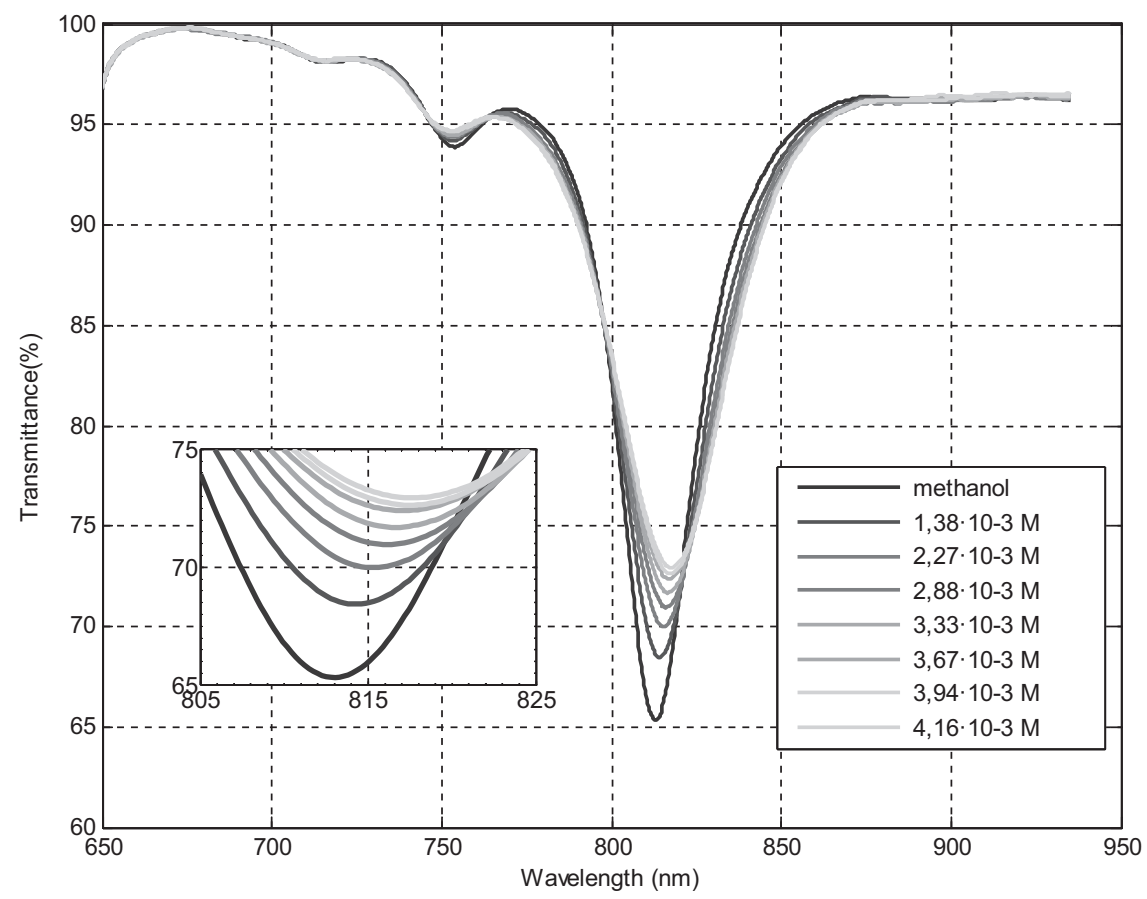

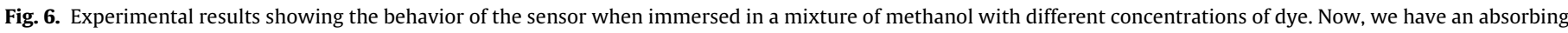
medium and the dips are not displaced, but reduced. In the inset we see an enlarged view of the area of the transmittance minima, to make this effect clearer. 
by the absorption we have chosen to represent one hundred minus the transmittance in percentage, taking this value as a measure of the "plasmon definition". The tendency is approximately linear and we can see how this value can clearly give us information about the concentration of the dye.

Summarizing, the principle of our method is proven by these measurements. First of all, media that are nonabsorbing for the wavelengths of plasmon resonance produce the expected common behavior of dip displacement when the refractive index increases. Since all substances are unavoidably absorbing for some wavelengths (and of course a mixture of methanol and ethylene glycol will definitely absorb for some wavelengths, but not for wavelengths around $800 \mathrm{~nm}$ ), what it is proven is that it is only when we tune plasmon resonances and absorption peaks when we observe the characteristic plasmon inhibition effect. This have been experimentally shown with our measurements, which, finally, confirm that we can use this mechanism as the basis of a presence sensor for IR-140 or any given absorbing compound (since the behavior is so clearly distinguishable between having or not an absorbing species in the methanol) and also as a measurement procedure to determine the concentration of the dye. In this way, without any need to add any recognizing agents, or to modify in any way the structure of conventional, nonselective SPR sensors, we can transform them into selective and we can use them for both measuring concentrations or presence of absorbing and nonabsorbing media.

\section{Conclusions}

As we have said, the method proposed by us in our work cited in reference 9 has been validated and the theoretical predictions made then and also in this paper have been experimentally confirmed, thus providing us with valuable basic information about the influence of absorption in the dynamics of surface plasma excitation in optical waveguides. This opens the way for the development of a new concept of operative sensors, based in very simple and convenient arrangements, that can provide the desired degree of selectivity only by choosing in the design stage the position of the plasmon resonance wavelengths to make them coincident to those where the analyte is absorbing. At the same time, we can work with the sensor in the usual way, so we can have simultaneously information on the variation of the real refractive index of the surrounding medium and on the presence of absorbing analytes in it. We can have then multiparameter measurements or a very easy way of measurement referencing.

With the same principle we can also measure the concentration of the absorbing species in a very easy way, and this procedure can be made very accurate if we have very well defined, narrow absorption peaks for the substances and tune very precisely our plasmon resonances to them.

Also, since one of the characteristics that are unique to asymmetrically deposited DLUWTs is that we can have multiple plasmon resonances we can conceive that for the same transducer we can detect two or more different absorbing substances and discriminate among them by properly tuning each of these resonances to characteristic absorption wavelengths. Or, we can design the system to have more than one coincidence for the same substance, thus providing us with a redundant information or permitting us to find the spectral mark of a given substance.

All these potentialities permit to predict the development of SPR based fiber microspectrometers consisting only of a conventional DLUWT, with all the advantages that it can present. Also, all the other possibilities of fiber sensors, like multiplexing, sensor juxtaposition and networks, remote detection remain open to exploit this concept as far as it can go, which will suppose a new line of development for operative chemical, biological and environmental sensors, small, cheap, robust, manageable and apt for in situ measurements.

\section{Acknowledgments}

This work has been partially supported by Spanish Ministry of Science and Innovation research project SPRINT (ref. CTQ200910550), by Community of Madrid research project FACTOTEM II (ref. S2009/ESP-1781) and by the European Social Fund and the European Fund for Regional Development.

\section{References}

[1] J. Homola, S.S. Yee, G. Gauglitz, Surface plasmon resonance sensors: review, Sensors and Actuators B 54 (1999) 3-15.

[2] J. Homola, Surface plasmon resonance sensors for detection of chemical and biological species, Chemical Review 108 (2008) 462-493.

[3] I. Abdulhalim, M. Zourob, A. Lakhtakia, Surface plasmon resonance for biosensing: a mini-review, Electromagnetics 28 (2008) 214-242.

[4] K. Kurihara, K. Suzuki, Theoretical understanding of an absorption based surface plasmon resonance sensor based on Kretchmann's theory, Analytical Chemistry 74 (2002) 696-701.

[5] A.A. Kolomenskii, P.D. Gershon, H.A. Schuessler, Surface-plasmon resonance spectrometry and characterization of absorbing liquids, Applied Optics 39 (2000) 3314-3320.

[6] A. Hanning, J. Roeraade, J.J. Delrow, R.C. Jorgenson, Enhanced sensitivity of wavelength modulated surface plamon resonance devices using dispersion from a dye solution, Sensors and Actuators B 54 (1999) 25-36.

[7] H. Komatsu, M. Miyachi, E. Fujii, D. Citterio, K. Yamada, Y. Sato, K. Kurihara, H. Kawaguchi, K. Suzuki, SPR sensor signal amplification based on dye-doped polymer particles, Science and Technology of Advanced Materials 7 (2006) $150-155$.

[8] M. Nakkach, P. Lecaruyer, F. Bardin, J. Sakly, Z. Ben Lakhdar, M. Canva, Absorption and related optical dispersion effects on the spectral response of a surface plasmon resonance sensor, Applied Optics 47 (2008) 6177-6182.

[9] Ó. Estéban, A. González-Cano, N. Díaz-Herrera, M.C. Navarrete, Absorption as a selective mechanism in surface plasmon resonance fiber optic sensors, Optics Letters 31 (21) (2006) 3089-3091.

[10] A. González-Cano, F.J. Bueno, Ó. Esteban, N. Díaz-Herrera, M.C. Navarrete, Multiple surface-plasmon resonance in uniform-waist tapered optical fibers with an asymmetric double-layer deposition, Applied Optics 44 (4) (2005) 519-526.

[11] Ó. Esteban, N. Díaz-Herrera, M.C. Navarrete, A. González-Cano, SPR sensors based on uniform-waist tapered fibers in reflective configuration, Applied Optics 45 (28) (2006) 7294-7298.

[12] M.C. Navarrete, N. Díaz-Herrera, A. González-Cano, Ó. Esteban, A polarizationindependent SPR fiber sensor, Plasmonics 5 (2010) 7-12.

[13] R. Gray, D. Walton, J. Bickerton, P. Richards, J. Heptinstall, The isomerisation of meso-amine substitude heptamethine dyes, Dyes and Pigments 30 (1996) 321-322.

[14] R. Weerasundam, G.G. Raju, An efficient algorithm for numerical computation of the complex dielectric permittivity using Hilber transform and FFT techniques through Kramers-Kronig relation, in: Proceedings of the 2004 International Conference on Solid Dielectrics, Toulouse (Francia), 5-9 July, 2004

[15] Ó. Esteban, M.C. Navarrete, A. González-Cano, E. Bernabeu, Simple model of compound waveguide structures used as fiber-optic sensors, Optics and Lasers in Engineering 33 (2000) 219-230.

\section{Biographies}

Ivo Leite was born in Porto (Portugal) in 1986. He is studying a Master degree in Physical Engineering in the University in Porto. He has made a stay in the Complutense University in Madrid where he developed a research in SPR fiber sensors within the Applied Optics Complutense Group as a part of his master.

María-Cruz Navarrete received her M.Sc. degree in Physics in 1986, and her Ph.D. degree of Physics in 1994 in the Universidad Complutense de Madrid (UCM, Spain). She joined Optics Department (UCM) in 1990 where she has carried out her research and teaching activities. She has been involved in fiber optic research and in several European and Spanish projects. During the last twelve years she has been working in optical fiber sensors, mainly applied to environmental measurements. Nowadays she is an assistant professor in the Optics Department and a member of the Applied Optics Complutense research group.

Natalia Díaz-Herrera received the M.Sc degree in physics from the Universidad Autónoma de Madrid, Madrid, Spain, in 2001 and the Ph.D. degree in applied optics from the Universidad Complutense de Madrid (UCM) in 2005. In 2006 she joined the Optics Department of the UCM as a lecturer, where she has carried out her research and teaching activities. She has been involved in several European and Spanish projects. Her research interests are optical fiber sensors, specifically for environmental applications. 
Agustín González-Cano was born in Madrid (Spain) in 1964. He received his M.Sc. in Physics (1987) and Ph.D. in Advanced Optics (1994) in the Universidad Complutense de Madrid (UCM), where he has developed his research and teaching activities during the last 22 years. Currently he is Professor in the School of Optics and Optometry of the UCM, where his main teaching subjects are Ophthalmic Optics, and Geometrical Optics. His research interests are basically optical sensors, and specifically fiber-optic sensors for environmental applications. In the past he has also been working in image processing, fringe pattern analysis and photoelasticity. In recent times he is also interested in research on the history of Optics. He has published more than 30 papers and has been involved in many different research projects, including some
European Union-funded ones. He is a member of the Spanish Optics Association (SEDOPTICA).

Óscar Esteban received his M.Sc. degree in Physics and the Ph.D. degree in Advanced Optics from Complutense University, Madrid, Spain, in 1997 and 2001 respectively. Since 2001, he has been with the University of Alcalá, Spain, where he is currently Associate Professor in the Electronics Department and Head of the Photonics Engineering Group (GRIFO). He is also a member of the Optical Society of America (OSA) His main research subjects are optical-fiber sensors, optical testing of materials, thin solid films and optoelectronics applications. 